\title{
Relationship between B-type natriuretic peptide serum level, echocardiographic TEI index and the degree of diastolic dysfunction in patients with heart failure with preserved systolic function
}

Danuta Karasek, Władysław Sinkiewicz, Jan Błażejewski

II Clinic of Cardiology, Nicolaus Copernicus University in Toruń, L. Rydygier Collegium Medicum in Bydgoszcz, University Hospital No 2 in Bydgoszcz, Poland

Submitted: 5 October 2009

Accepted: 21 December 2009

Arch Med Sci 2011; 7, 3: 449-456

DOI: 10.5114/aoms.2011.23411

Copyright (C) 2011 \& Termedia

\section{Abstract}

Introduction: The growing number of heart failure (HF) patients is becoming an important issue in cardiology. B-type natriuretic peptide (BNP) is a recognized marker of HF, including in patients with preserved systolic function. The TEI index is an indicator of left ventricular function. The aim of the study was to evaluate the relationship between BNP serum level, TEI index and the degree of diastolic dysfunction in patients with HF symptoms and preserved systolic function.

Material and methods: Hundred patients with arterial hypertension and preserved systolic function were enrolled in the study. The study group consisted of 51 individuals with impaired diastolic function and HF symptoms. Fourty-nine hypertensive individuals without HF symptoms were assigned to the control group. B-type natriuretic peptide and echocardiographic examination were performed. Patients were divided into 4 subgroups - with normal diastolic function, impaired relaxation, pseudonormalization and restriction.

Results: Median value of BNP in patients with normal diastolic function was $28.36 \mathrm{pg} / \mathrm{ml}, 87.10 \mathrm{pg} / \mathrm{ml}$ in patients with impaired relaxation, $212.75 \mathrm{pg} / \mathrm{ml}$ and $461.56 \mathrm{pg} / \mathrm{ml}$ in the pseudonormalization and restriction group respectively $(p<0.0001)$. The median value of the TEI index was: 0.386 in patients with normal diastolic function, 0.507 in individuals with impaired relaxation, 0.639 and 0.725 in the pseudonormalization and restriction group respectively. All the differences were statistically significant $(p<0.0001)$. A significant positive correlation $(r=0.80, p<0.001)$ between BNP and the TEl index was found.

Conclusions: In hypertensive patients with HF symptoms and preserved left ventricular systolic function a highly significant increase in BNP serum level and in the TEI index values related to the deterioration of diastolic dysfunction was found.

Key words: B-type natriuretic peptide, TEl index, heart failure with preserved systolic function.

\section{Introduction}

Heart failure (HF) is becoming a more and more serious problem of contemporary cardiology. Heart failure as an inability to maintain sufficient cardiac output may be a result of either impaired systolic function and/or abnormal ventricular filling. This provides the basis for distinguishing (according to ESC guidelines) systolic heart failure and heart failure with
Corresponding author:

Danuta Karasek MD, PhD

University Hospital No 2

75 Ujejskiego

85-168 Bydgoszcz, Poland Phone/fax: +48 523655653

E-mail: karasek@op.pl 
normal ejection fraction (HFNEF). It is estimated that $30-50 \%$ of patients with HF suffer from HFNEF $[1,2]$. The crucial feature of this kind of HF is impaired ventricular filling resulting in maintaining cardiac output at the expense of elevated filling pressure. One of the most frequent causes of HFNEF is hypertension. A fundamental mechanism leading to heart failure development in the course of hypertension is ventricular pressure overload.

Considering the importance of the issue, early diagnosis is crucial for patients with HF. It is diagnosed in the presence of coexistent heart failure symptoms and objective features of systolic or diastolic dysfunction. To date there is no single test that is a diagnostic criterion for $\mathrm{HF}$ [3]. Echocardiography is a preferred method; biochemical markers with special regard to natriuretic peptides, especially type B (BNP), are also of great importance. However, in populations with low risk of left ventricular dysfunction the determination of these markers often gives false positive results and requires further verification [4]. It is expected that the evaluation of BNP together with comprehensive echocardiographic examination will allow us to single out individuals with cardiac output maintained through the elevation of ventricular filling pressure. These patients already have symptoms of HF or are at high risk of developing heart failure in the near future [3].

A quick evaluation of left ventricle function by means of echocardiography may be done by calculating the myocardial performance index (MPI), also known as the TEI index, which reflects both left ventricular systolic and diastolic function [5]. A normal TEI index should be below 0.4 and its increasing values are related to deteriorated left ventricular function.

The majority of studies investigating chronic heart failure concern systolic HF, but a growing number of patients diagnosed with HFNEF can be observed. Therefore the present study was designed to evaluate the relationship between BNP serum level, echocardiographic TEl index and the degree of diastolic dysfunction in hypertensive patients with HF symptoms and preserved systolic function. It was assumed that the concomitant evaluation of these parameters may improve the accuracy of HFNEF diagnosis.

\section{Material and methods}

Hundred patients with hypertension diagnosed at least 5 years earlier, sinus rhythm and preserved systolic function (LVDd $<6.0 \mathrm{~cm}$, LVEF $>45 \%$ ) were enrolled in the study. Patients with ischaemic heart disease, systolic HF, severe valvular heart disease, primary cardiomyopathy and constrictive pericarditis were excluded from the study. All participants of the study signed the informed consent form. The study population was divided into two groups: one with heart failure symptoms from NYHA class II to IV (51 patients; 4 males and 47 females with a mean age of $65.9 \pm 10.1$ years) and a control group of 49 patients without HF symptoms. A comparative analysis of both groups found no significant differences in terms of age, sex, diabetes mellitus, hyperlipidaemia, obesity, smoking or family history of HF. All patients received optimal pharmacotherapy according to valid standards.

Venous blood samples were taken by venipuncture in the antecubital region with minimal venostasis, between $7.00 \mathrm{am}$ and $9.00 \mathrm{am}$ after a 30-min rest, in a sitting position. No longer than 45 min elapsed between blood sampling and measurement. B-type natriuretic peptide serum level determination was performed by means of microparticle enzyme immunoassay (MEIA) AxSYM BNP test by the Abbott Diagnostics Division (normal values $<100 \mathrm{pg} / \mathrm{ml}$ ) [6].

Detailed echocardiographic examination using a transthoracic 2.5 MHz probe of Vivid 7.0 (General Electric Company) together with simultaneous ECG recording was performed on the day of the BNP serum level measurement. Examination comprised mono- and two-dimensional echocardiography, conventional and colour Doppler flow imaging (pulsed- and continuous wave Doppler) and tissue Doppler echocardiography. All of the chambers' dimensions and wall thicknesses were measured. Systolic function was evaluated both by assessment of regional contractility disturbances and by calculation of left ventricular ejection fraction (LVEF) by means of the biplane Simpson method. Valvular structure and function together with intracardiac blood flow were also evaluated.

After excluding left ventricular enlargement $($ LVEDd $\leq 6.0 \mathrm{~cm})$, contractility disturbances and haemodynamically significant valvular disease, and after confirming normal ejection fraction (LVEF $\geq 45 \%$ ), detailed left ventricular diastolic function assessment together with the TEI index calculation was performed. For diastolic function evaluation Doppler techniques of mitral inflow and pulmonary vein flow estimation were used together with the tissue Doppler imaging of mitral annulus velocity, which, according to Feigenbaum, are of the greatest value in modern clinical practice [7]. After detailed echocardiographic examination all patients were divided into four groups according to the Canadian Cardiovascular Society diastolic dysfunction classification from 1996 (Table I) [8].

For normally distributed variables the mean value and standard deviation were calculated. For variables not normally distributed the median value and lower and upper quartile were determined. Pairs of variables were compared with the MannWhitney $U$ test. Kruskal ANOVA was used to 
Table I. Canadian Cardiovascular Society diastolic dysfunction classification from 1996

\begin{tabular}{|c|c|c|c|c|c|}
\hline Degree of diastolic dysfunction & $\mathrm{E} / \mathrm{A}$ & $\mathrm{dtE}$ & $S / D$ & $\operatorname{Ar}$ & Ar-A \\
\hline Normal & $1-2$ & $150-200$ & $\geq 1$ & $<0.35$ & $<20$ \\
\hline Mild (Impaired relaxation) & $<1$ & $>200$ & $\geq 1$ & $<0.35$ & $<20$ \\
\hline Moderate (Pseudonormalisation) & $1-2$ & $150-200$ & $0.5-1$ & $\geq 0.35$ & $\geq 20$ \\
\hline Severe (Restriction) & $>2$ & $<150$ & $<0.5$ & $\geq 0.35$ & $\geq 20$ \\
\hline
\end{tabular}

E/A - ratio of early (E) to late atrial (A) mitral peak flow velocity, dtE - early mitral flow deceleration time, S/D - ratio of pulmonary venous systolic (S) to diastolic (D) peak flow, Ar-peak reverse flow velocity at atrial contraction, Ar-A-difference between pulmonary venous and mitral A wave duration

Table II. Clinical profile of study and control group

\begin{tabular}{|c|c|c|c|c|c|}
\hline Degree of diastolic dysfunction & $E / A$ & $\mathrm{dtE}$ & $S / D$ & $\mathrm{Ar}$ & Ar-A \\
\hline Normal & $1-2$ & $150-200$ & $\geq 1$ & $<0.35$ & $<20$ \\
\hline Mild (Impaired relaxation) & $<1$ & $>200$ & $\geq 1$ & $<0.35$ & $<20$ \\
\hline Moderate (Pseudonormalisation) & $1-2$ & $150-200$ & $0.5-1$ & $\geq 0.35$ & $\geq 20$ \\
\hline Severe (Restriction) & $>2$ & $<150$ & $<0.5$ & $\geq 0.35$ & $\geq 20$ \\
\hline
\end{tabular}

compare several independent samples. To define the relationship between the studied parameters the Spearman correlation coefficient was calculated. The significance level was set at $p<0.05$ for all tests. The results were worked out using StatSoft's Statistica 6.0.

\section{Results}

\section{Clinical characteristics of study and control group}

Among study participants 25 patients were in NYHA class II, 19 patients in NYHA class III and 7 patients in NYHA class III/IV (Table II).

\section{Results of laboratory tests and echocardiographic examination}

The comparison of ejection fraction and diastolic parameters calculated after echocardiographic examination was conducted in the study and control group. Analysis of these parameters revealed significantly higher values of diastolic dysfunction indices: dtE, IVRT, $\operatorname{Ar}, \operatorname{Ar}(\mathrm{t})-\mathrm{A}(\mathrm{t})$, E/E' ratio and TEl index together with lower values of S/D in patients from the study group compared to controls. Another observation worth mentioning is the lack of a statistically significant difference in the commonly used E/A ratio between the study and control group. All parameters are presented in Table III.

After echocardiographic examination patients were divided into 4 groups depending on the degree of diastolic dysfunction. Diastolic dysfunction was graded according to a useful and universally applied classification (CCS guidelines 1996) based on mitral inflow parameters and pulmonary vein flow evaluation (Table I). Impaired relaxation accounts for mild diastolic dysfunction, pseudonormalisation profile for moderate dysfunction, while restriction corresponds to severe diastolic dysfunction according to Canadian criteria. Along with diastolic function deterioration a statistically significant increase of BNP serum level $(p<0.001)$ and TEI index value $(p<0.0001)$ was observed together with exacerbation of heart failure symptoms assessed with NYHA classification (Table IV).

\section{Correlation between studied parameters}

A significant positive correlation between BNP serum levels and the TEI index with correlation coefficient $r=0.80$ ( $p<0.0001)$ was observed in the whole study population.

Table III. Comparison of echocardiographic parameters between study and control group

\begin{tabular}{|lccc|}
\hline $\begin{array}{l}\text { Parameter } \\
\text { [unit] }\end{array}$ & $\begin{array}{c}\text { Study group } \\
(n=51)\end{array}$ & $\begin{array}{c}\text { Control group } \\
(n=49)\end{array}$ & Value of $p$ \\
\hline EF [\%] & $60 \pm 5$ & $67 \pm 8$ & NS \\
\hline dtE $[\mathrm{ms}]$ & $207 \pm 47$ & $190 \pm 16$ & 0.02 \\
\hline IVRT [ms] & $106 \pm 23$ & $96 \pm 7$ & 0.02 \\
\hline E/A & $0.91 \pm 0.55$ & $0.95 \pm 0.19$ & NS \\
\hline E'/A' & $0.68 \pm 0.14$ & $1.03 \pm 0.32$ & $<0.0001$ \\
\hline E/E' & $12.7 \pm 7.4$ & $9.15 \pm 2.7$ & $<0.0001$ \\
\hline S/D & $1.1 \pm 0.3$ & $1.44 \pm 0.2$ & $<0.0001$ \\
\hline Ar [m/s] & $0.31 \pm 0.03$ & $0.29 \pm 0.02$ & 0.0003 \\
\hline Ar(t)-A(t) [ms] & $-1.5 \pm 2.4$ & $-17.01 \pm 1.7$ & $<0.0001$ \\
\hline TEl & $0.569 \pm 0.12$ & $0.391 \pm 0.05$ & $<0.0001$ \\
\hline
\end{tabular}

$E^{\prime} / A^{\prime}$ - ratio of peak early to late diastolic velocity of the mitral annulus, E/E' ratio of mitral peak velocity of early diastolic filling to peak early diastolic velocity of the mitral annulus, IVRT - isovolumic relaxation time 
Table IV. Comparison of BNP serum level, TEl index between subgroups with different diastolic function profile and distribution of patients with NYHA class I, II, III and III/IV in these subgroups

\begin{tabular}{|c|c|c|c|c|c|}
\hline \multirow[t]{2}{*}{ Parameter [unit] } & \multicolumn{4}{|c|}{ Diastolic function } & \multirow[t]{2}{*}{ Value of $p$} \\
\hline & $\begin{array}{l}\text { Normal } \\
(n=46)\end{array}$ & $\begin{array}{l}\text { Impaired relaxation } \\
\qquad(n=31)\end{array}$ & $\begin{array}{l}\text { Pseudonormalisation } \\
\qquad(n=16)\end{array}$ & $\begin{array}{l}\text { Restriction } \\
\quad(n=7)\end{array}$ & \\
\hline BNP $[\mathrm{pg} / \mathrm{ml}]$ & $28.36 \pm 18.17$ & $87.10 \pm 37.90$ & $212.75 \pm 93.02$ & $461.56 \pm 202.1$ & $<0.0001$ \\
\hline TEI & $0.386 \pm 0.035$ & $0.507 \pm 0.071$ & $0.639 \pm 0.094$ & $0.725 \pm 0.096$ & $<0.0001$ \\
\hline \multicolumn{6}{|l|}{ NYHA [\%] } \\
\hline I & 92 & 8 & 0 & 0 & \\
\hline II & 8 & 86 & 6 & 0 & \\
\hline III & 0 & 6 & 94 & 0 & \\
\hline III/IV & 0 & 0 & 0 & 100 & \\
\hline
\end{tabular}
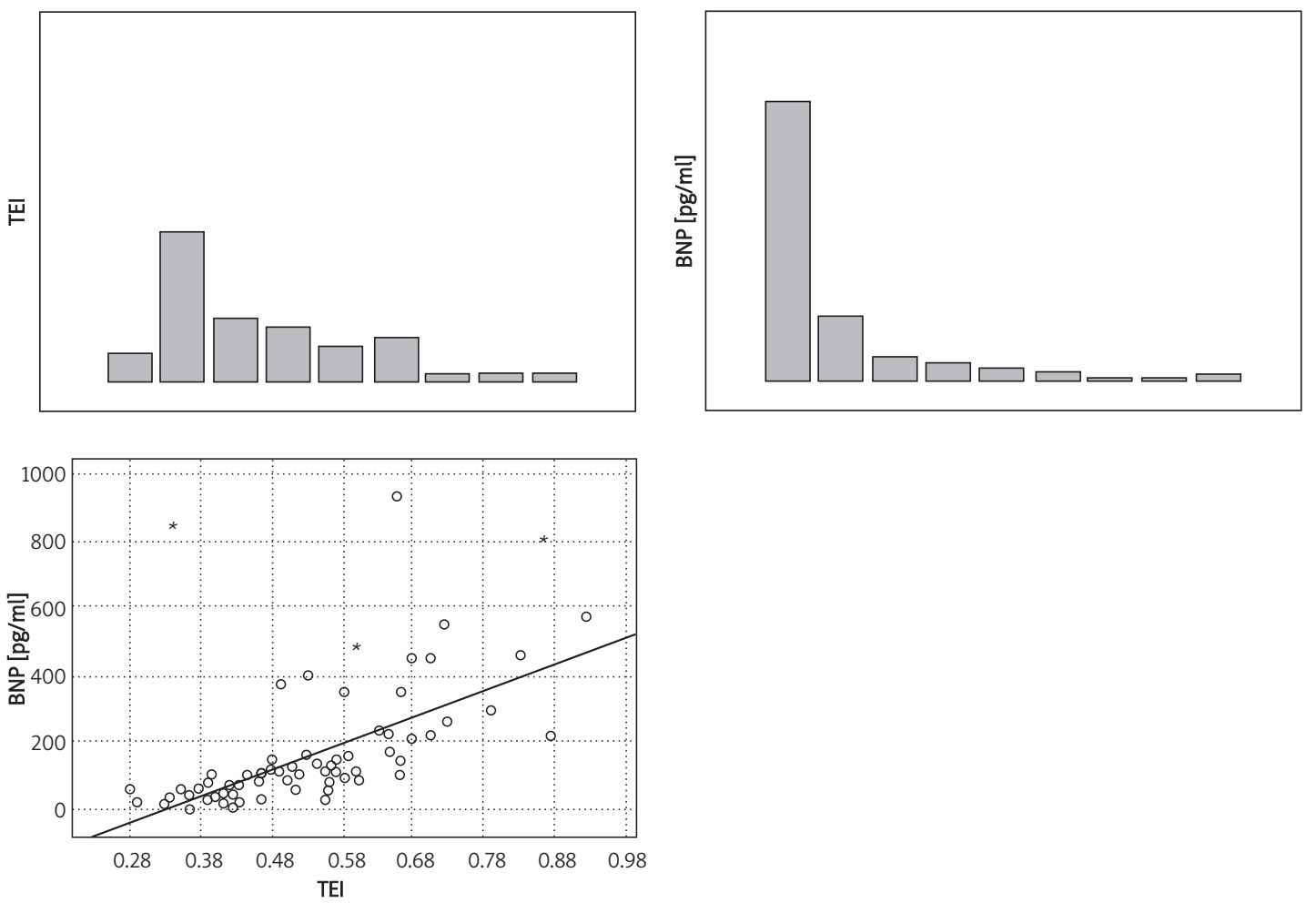

Figure 1. Correlation between BNP serum level and TEI index values in the study population

\section{Discussion}

An important role of neurohormonal activation in heart failure has been emphasized in recent years. Advanced research led to the discovery of multiple substances whose serum levels were found to be elevated in the course of HF. Among them BNP seems to have an exceptional position. This neurohormone is released from ventricular myocardium in response to volume or pressure overload. Its secretion is induced mainly by myocardial wall strain or ischaemia. A lot of randomized trials concerning mostly systolic heart failure patients have proved that increased BNP serum level allows for a reliable diagnosis of HF [9].
Markedly fewer publications concern BNP serum level in patients with HFNEF. The majority of them underline a statistically significant, though less spectacular, increase of BNP concentration in individuals with HF symptoms and left ventricular diastolic dysfunction compared to healthy controls $[10,11]$.

In The Breathing Not Properly Multinational Study in 1,586 patients referred to hospital for sudden dyspnoea BNP serum level was measured together with a detailed echocardiographic examination. Four hundred and fifty-two patients were diagnosed with heart failure including 165 (36.5\%) with isolated diastolic dysfunction (LVEF > 45\%). 
Maisel et al. summarizing the results of the study proved that patients with HF had higher BNP concentration than individuals without heart failure symptoms. Heart failure with normal ejection fraction was, however, associated with lower BNP serum levels than systolic dysfunction $(413 \mathrm{pg} / \mathrm{ml}$ vs. $821 \mathrm{pg} / \mathrm{ml}$ ). The BNP value of $100 \mathrm{pg} / \mathrm{ml}$ allowed for a diagnosis of systolic HF and HFNEF with $86 \%$ and $75 \%$ sensitivity respectively [12].

Similar results were obtained by Bursi et al. after the echocardiographic evaluation of ejection fraction (EF) and BNP serum level measurement in 556 patients with HF. Two hundred and fourty-two patients (44\%) with isolated diastolic dysfunction (LVEF > 50\%) and significantly increased BNP level were singled out. However, the highest BNP concentration was observed in individuals with diastolic dysfunction combined with reduced left ventricular ejection fraction [13]. Parallel findings come from other studies performed on smaller populations in Great Britain and China [14, 15].

Tschöpe et al. conducted another study involving patients with HF symptoms and isolated diastolic dysfunction. The mean BNP serum level in chronic heart failure (CHF) patients was $189.54 \mathrm{pg} / \mathrm{ml}$ and was remarkably higher than in healthy controls $51.89 \mathrm{pg} / \mathrm{ml}(p<0.001)$. Moreover, a statistically significant relationship between BNP serum level and the degree of diastolic dysfunction evaluated by means of echocardiography was observed. Another correlation was found between BNP concentration and end-diastolic left ventricular pressure measured invasively. The authors also emphasized the high negative predictive value (94\%) of BNP serum level determination for the diagnosis of HF [16].

Recent reports also underline the remarkable value of BNP serum level determination for diagnosis and evaluation of disease advancement in patients with HF including those with preserved systolic function $[17,18]$.

The results of all the above-mentioned research are in concordance with the present study, whose significant finding is that BNP serum level is markedly elevated in patients with HF symptoms and preserved systolic function and that the elevation is proportional to the intensity of diastolic dysfunction in echocardiography. It is probably because increasing diastolic dysfunction is associated with increasing left ventricle volume overload. Contrary observations were made by Dahlström, who found only insignificant differences in BNP serum levels between patients with mild diastolic dysfunction evaluated by means of echocardiography and individuals with normal diastolic function. The dissimilar findings may be a result of a different study population as Dahlström enrolled asymptomatic patients or individuals with symptoms non-specific for HF [19].
Lukowicz et al. investigating a group of 1,678 patients found statistically significant differences in BNP serum level between patients with diastolic dysfunction and healthy controls $(20.3 \pm 4.7 \mathrm{pg} / \mathrm{ml}$ vs. $9.6 \pm 0.5 \mathrm{pg} / \mathrm{ml}, p<0.001)$. At the same time more pronounced elevation was observed in individuals with myocardial hypertrophy $(37.3 \pm 49.1 \mathrm{pg} / \mathrm{ml}$, $p<0.001$ ). It was underlined that the more advanced the diastolic dysfunction, the more increased BNP concentration was noted. The authors also emphasize that BNP level within the normal range allows one to exclude diastolic dysfunction and concomitant myocardial hypertrophy with $99.9 \%$ probability. However, there were also a few cases with very low BNP concentration $(9.7 \pm 1.7 \mathrm{pg} / \mathrm{ml})$ in spite of the impaired relaxation on echocardiography [20]. It could be a result of conducting the study in a general population without regard to their symptoms. In this context, it is worth mentioning that in our study the investigated group was divided according to the presence of HF symptoms.

The present study proved that increasing diastolic dysfunction is associated with more severe heart failure symptoms assessed by means of NYHA classification. Among individuals with impaired relaxation the majority of patients were in NYHA class II (86\%), in the pseudonormalisation group most patients were in NYHA class III (94\%), while restriction was associated with the most severe heart failure - all patients were in NYHA class III/IV (100\%). A statistically significant increase of TEI index and BNP serum level connected with more advanced heart failure should also be underlined. Similar findings were reported by Wieczorek et al., who determined BNP serum level in 1050 patients with HF symptoms and healthy volunteers in order to define the threshold value for heart failure. In healthy participants the mean BNP value was $9.29 \mathrm{pg} / \mathrm{ml}$, in patients with $\mathrm{HF}$ symptoms BNP serum level depended on NYHA class and came to $83.1 \mathrm{pg} / \mathrm{ml}(49.4-137 \mathrm{pg} / \mathrm{ml})$ in NYHA class I, $235 \mathrm{pg} / \mathrm{ml}$ (137-391 pg/ml) in NYHA II, $459 \mathrm{pg} / \mathrm{ml}(200-871 \mathrm{pg} / \mathrm{ml})$ in NYHA III and $1119 \mathrm{pg} / \mathrm{ml}$ $(728-1300 \mathrm{pg} / \mathrm{ml})$ in NYHA class IV. Setting the criterion for heart failure diagnosis at $100 \mathrm{pg} / \mathrm{ml}$ the authors reported $82 \%$ sensitivity and $99 \%$ specificity [21]. In this study, however, there was no distinction between patients with systolic heart failure and HFNEF. A correlation between BNP serum level and NYHA functional class was also documented by Caboral et al. The authors also indicated the possibility for monitoring results of ambulatory treatment of HF patients by means of BNP determination, since it seems to be a more objective indicator of clinical state than NYHA classification [22].

Parthenakis et al. evaluated the TEI index and other echocardiographic indices of left ventricular 
function together with maximal oxygen uptake in ergospirometry in 42 heart failure patients in NYHA class II to IV and with LVEF $<40 \%$. The investigators proved that the more advanced the heart failure assessed by NYHA classification, the higher the TEI index $(0.59 \pm 0.1$ in NYHA class II and $1.06 \pm 0.24$ in NYHA class III/IV) - all differences were statistically significant at $p<0.001$ [23].

The TEI index is defined as the ratio of isovolumic contraction time plus isovolumic relaxation time to left ventricular ejection time and is a useful non-invasive parameter of global, both systolic and diastolic, left ventricular function. Its most prominent values are reproducibility, simplicity of the measurement and independence of heart rate, blood pressure or pre-load. It was proved in multiple clinical trials that the TEI index is strongly positively correlated with ejection fraction and left ventricular end-diastolic pressure in patients with heart failure [24].

The present study indicates a statistically significant elevation of the TEl index in HF patients with normal EF, which was more distinct in patients with more advanced diastolic dysfunction. This is in concordance with the findings of Zhang et al. from a study evaluating E/A, dtE, TEI index and haemodynamic indices from heart catheterisation. The authors described a positive correlation between TEI index and left ventricular mean diastolic pressure (LVMDP; $r^{2}=0.63, p<0.0001$ ). TEI index $\geq 0.65$ had $82 \%$ sensitivity and $96 \%$ specificity in diagnosing pseudonormalisation or restriction [25]. Similarly, other authors have noted significantly higher values of TEI index in patients with more advanced diastolic dysfunction [26, 27].

The usefulness of the TEI index in the assessment of diastolic dysfunction was also a subject of an investigation conducted by Bruch et al. They performed echocardiographic examination with TEI index calculation in 107 study participants 36 healthy volunteers, 36 patients with normal systolic function and 35 individuals with systolicdiastolic heart failure. TEI index values were significantly higher in patients with diastolic dysfunction compared to healthy controls $(0.53 \pm 0.14$ vs. $0.39 \pm 0.07, p<0.05)$. The highest levels of TEl index were observed in patients with systolic-diastolic HF $(0.94 \pm 0.43, p<0.01)$ [28].

In the present study increased TEI index was noted in early stages of diastolic dysfunction, which correlated positively with other parameters of left ventricular diastolic function. It is probably connected with prolonged isovolumic diastole a "diastolic" component - in patients with impaired relaxation of the left ventricle. It cannot be excluded that a statistically significant increase of the TEI index in patients with more advanced diastolic dysfunction may be partially a result of latent systolic dysfunction. In the studied population there was no confirmed systolic dysfunction and LVEF was within the normal range. However, with regard to frequent left ventricular hypertrophy in this group of patients this mechanism seems highly plausible.

In our study there is a strong positive correlation between BNP serum level and the TEI index value $(r=0.80, p<0.0001)$. This relationship may be a result of increasing pressure load of the left ventricle in patients with more advanced diastolic dysfunction associated with growing end-diastolic pressure leading to BNP hypersecretion. Moreover, a plausible mechanism related to latent systolic dysfunction should also be considered, as it also contributes to increase of both TEI index and BNP serum level.

Similar results were reported by Tanabe et al., who measured BNP serum concentration in 74 patients with various cardiovascular conditions and correlated the values with the echocardiographic TEl index. Both BNP and TEl index values were markedly increased in the group with confirmed diastolic dysfunction compared to those without diastolic impairment. The highest levels of both studied parameters were found in patients with systolic HF (LVEF < 50\%). In patients with TEI $\geq 0.45$ the BNP serum level was significantly increased compared to individuals with TEI $<0.45$ (241.4 $\pm 451.2 \mathrm{pg} / \mathrm{ml}$ vs. $65.9 \pm 81.8 \mathrm{pg} / \mathrm{ml}, p<0.05)$ and inversely, in a group with BNP $\geq 73 \mathrm{pg} / \mathrm{ml}$ TEl index values were significantly higher compared to patients with BNP $<73 \mathrm{pg} / \mathrm{ml}(0.57 \pm 0.24$ vs. 0.46 $\pm 0.17, p<0.05)$ [29].

Okawa et al. reported significantly higher TEI index values in patients with hypertrophic cardiomyopathy compared to the control group (0.55 \pm 0.12 vs. $0.36 \pm 0.08, p<0.0001)$. They also found a positive correlation between TEl index and BNP serum level $(r=0.61, p<0.0001)$. In multiregression analysis TEI index and E/A turned out to be independent predictors of increased BNP serum level [30].

It should be underlined that in the literature there are only a few analyses of the relationship between BNP serum concentration and echocardiographic parameters including the TEI index in patients with HFNEF. Our study proved a strong positive correlation between these parameters. The remarkable diagnostic value of BNP serum level determination and TEl index calculation in patients with diastolic dysfunction may be an important hint for clinicians to use concomitant measurement of these parameters in order to improve the accuracy of HFNEF diagnosis, especially in the early oligosymptomatic stages of the disease. Moreover, with regard to the fact that the TEl index is a parameter reflecting both systolic and diastolic function, and splitting these components is difficult, 
it may be treated as a screening test allowing for identification of patients with marked left ventricular dysfunction. In these patients, with preserved systolic function and elevated TEI index, latent systolic dysfunction should also be considered. The present study is an attempt to create a simple algorithm for early and objective diagnosis of heart failure, including HF with preserved systolic function, that could improve treatment of patients in this asymptomatic stage of the disease.

In conclusion, in hypertensive patients with heart failure symptoms and preserved left ventricular systolic function a statistically significant increase of BNP serum level and the TEl index occurs along with the deterioration of left ventricular diastolic function on echocardiography. B-type natriuretic peptide concentration and TEI index values in patients with diastolic HF are strongly positively correlated with each other. Concomitant measurement of these parameters may constitute a valuable supplement to routine examinations in patients with HF symptoms and preserved systolic function and considerably improve the accuracy of diastolic heart failure diagnosis.

\section{References}

1. Grigorian SL, Gonzalez-Juanatey JR, Roman AV, et al. The death rate among hospitalized heart failure patients with normal and depressed left ventricular ejection fraction in the year following discharge: evolution over a 10-year period. Eur Heart J 2005; 26: 2251-8.

2. Hunt SA, Abraham WT, Chin MH, et al. ACC/AHA 2005 Guideline Update for the Diagnosis and Management of Chronic Heart Failure in the Adult-Summary Article A Report of the American College of Cardiology/American Heart Association Task Force on Practice Guidelines. J Am Coll Cardiol 2005; 46: 1116-43.

3. Doust JA, Pietrzak E, Dobson A, et al. How well does B-type natriuretic peptide predict death and cardiac events in patients with heart failure: systematic review. BMJ 2005; 330: 625.

4. Redfield MM, Rodeheffer RJ, Jacobsen SJ, et al. Plasma brain natriuretic peptide to detect preclinical ventricular systolic or diastolic dysfunction: a community-based study. Circulation 2004; 109: 3176-81.

5. Tei C, Ling LH, Hodge DO, et al. New index of combined systolic and diastolic myocardial performance: a simple and reproductible measure of cardiac function - a study in normals and dilated cardiomyopathy. J Cardiol 1995; 2: 357-66.

6. Textbook of AxSYM BNP Abbott System No REF 8G82-20 test. 2005

7. Feigenbaum H, Armstrong WF, Ryan T. Echokardiografia. Lippincott Williams \&Wilkins 2005; 173-84.

8. Rakowski H, Appelton CP, Chan KL, et al. Canadian consensus recommendations for the measurement and reporting of diastolic dysfunction by echocardiography: from the Investigators of Consensus on Diastolic Dysfunction by Echocardiography. J Am Soc Echocardiogr 1996; 9: 736.

9. McDonagh TA. Asymptomatic left ventricular dysfunction in the community. Curr Cardiol Rep 2000; 2: 470-4.
10. Nakao S, Goda A, Yuba M, et al. Characterisation of left ventricular filling abnormalities and its relation to elevated plasma brain natriuretic peptide level in acute to chronic diastolic heart failure. Circulation J 2007; 71: 1412-7.

11. Grewal J, McKelvie R, Lonn E, et al. BNP and NT-proBNP predict echocardiographic severity of diastolic dysfunction. Eur J Heart Fail 2008; 10: 252-9.

12. Maisel AS, McCord J, Nowak RMJ, et al. Bedside B-type natriuretic peptide in the emergency diagnosis of heart failure with reduced or preserved ejection fraction. Results from the Breathing Not Properly Multinational Study. Am Coll Cardiol 2003; 41: 2010-7.

13. Bursi F, Weston SA, Redfield MM, et al. Systolic and diastolic heart failure in the community. JAMA 2006; 296: 2209-16.

14. Thomas MD, Fox KF, Wood DA, et al. Echocardiographic features and brain natriuretic peptides in patients presenting with heart failure and preserved systolic function. Heart 2006; 92: 603-8.

15. Wei T, Zeng C, Chen L, et al. Systolic and diastolic heart failure are associated with different plasma levels of B-type natriuretic peptide. Int J Clin Pract 2005; 59: 891-4.

16. Tschöpe C, Kasner M, Westermann D, et al. The role of NT-proBNP in the diagnostics of isolated diastolic dysfunction: correlation with echocardiographic and invasive measurements. Eur Heart J 2005; 26: 2277-84.

17. Martos R, Baugh J, Ledwidge M, et al. Diagnosis of heart failure with preserved ejection fraction: improved accuracy with the use of markers of collagen turnover. Eur J Heart Fail 2009; 11: 191-7.

18. Troughton RW, Richards AM. B-type natriuretic peptides and echocardiographic measures of cardiac structure and function. J Am Cool Cardiol Img 2009; 2: 216-25.

19. Dahlström U. Can natriuretic peptides be used for the diagnosis of diastolic heart failure? Eur J Heart Fail 2004; 6: 281-7.

20. Lukowicz TV, Fischer M, Hense HW, et al. BNP as a marker of diastolic dysfunction in the general population: Importance of left ventricular hypertrophy. Eur J Heart Fail 2005; 7: 525-31.

21. Wieczorek SJ, Wu A, Christenson R. A rapid B-type natriuretic peptide assay accurately diagnoses left ventricular dysfunction and heart failure: a multicenter evaluation. Am Heart J 2002; 144: 834-9.

22. Caboral M, Mitchell J. B-type natriuretic peptide: a new tool in the armamentarium used to accurately diagnose heart failure. Prog Cardiovasc Nurs 2003; 18: 190-3.

23. Parthenakis FI, Kanakaraki MK, Kanoupakis EM, et al. Value of Doppler index combining systolic and diastolic myocardial performance in predicting cardiopulmonary exercise capacity in patients with congestive heart failure. Chest 2002; 121: 1935-41.

24. Sutton MJ, Wiegers SE. The Tei index - a role in the diagnosis of heart failure? Eur Heart J 2000; 21: 1822-4.

25. Zhang $\mathrm{H}$, Otsuji $\mathrm{Y}$, Matsukida K, et al. Noninvasive differentiation of normal from pseudonormal/restrictive mitral flow using TEI index combining systolic and diastolic function. Circ J 2002; 66: 831-6.

26. Su HM, Lin TH, Voon WC, et al. Differetiation of left ventricular diastolic dysfunction, identification of pseudonormal/restrictive mitral inflow pattern and determination of left ventricular filling pressure by Tei index obtained from tissue Doppler echocardiography. Echocardiography 2006; 23: 287-94.

27. Keser N, Yildiz S, Kurto N, et al. Modiifed TEI index: a promising parameter in essential hypertension? Echocardiography 2005; 22: 296-304. 
28. Bruch C, Gradaus R, Gunia S, et al. Doppler tissue analysis of mitral annular velocities: evidence for systolic abnormalities in patients with diastolic heart failure. J Am Soc Echocardiogr 2003; 16: 1031-6.

29. Tanabe K, Ono M, Asanuma T, et al. Doppler echocardiography - derived index of myocardial performance (TEl index): comparison with brain natriuretic peptide levels in various hart disease. Jpn Circ J 2001; 65: 637-42.

30. Okawa M, Kitaoka H, Matsumura Y, et al. Functional assessment by myocardial performance index (Tei index) correlates with plasma brain natriuretic peptide concentration in patients with hypertrophic cardiomyopathy. Circ J 2005; 69: 951-7. 